\title{
Improving the Performance of Parallel LISP by Compile Time Analysis
}

\author{
Jürgen Knopp \\ Siemens AG, Otto Hahn Ring 6 \\ 8000 München 83, Germany \\ email:jk@km21.zfe.siemens.de
}

\begin{abstract}
This paper presents a method to eliminate parts of the overhead introduced by parallel constructs in EDS LISP, a parallel extension of Common Lisp. Futures support a declarative style of explicit parallel programming. In languages with futures there is no static distinction between data calculated locally and data calculated by another process. Hence before data can be accessed a dynamic accessibility check has to be done. If the data is not yet determined an implicit wait has to be performed. Checks are done even when all data objects are present.

An algorithm is presented which does away with a significant amount of these checks. It is a variant of (intra-procedural) abstract interpretation, up to some extend similar to strictness analysis. Unlike other approaches the presented approach copes with both functional and non-functional LISP. The algorithm has been implemented in Common Lisp. The benchmarks are promising.
\end{abstract}

\section{EDS LISP: Programming with Futures}

The EDS Lisp (see [HaHe 90]) language supports explicit large grain parallelism. Aside from message passing and synchronization mechanisms the main language extension w.r.t. Common Lisp is the future. This construct is borrowed with some changes from other parallel Lisp dialects ([Hal 85]). We first show a (somewhat naive) parallel variant of quicksort using futures:

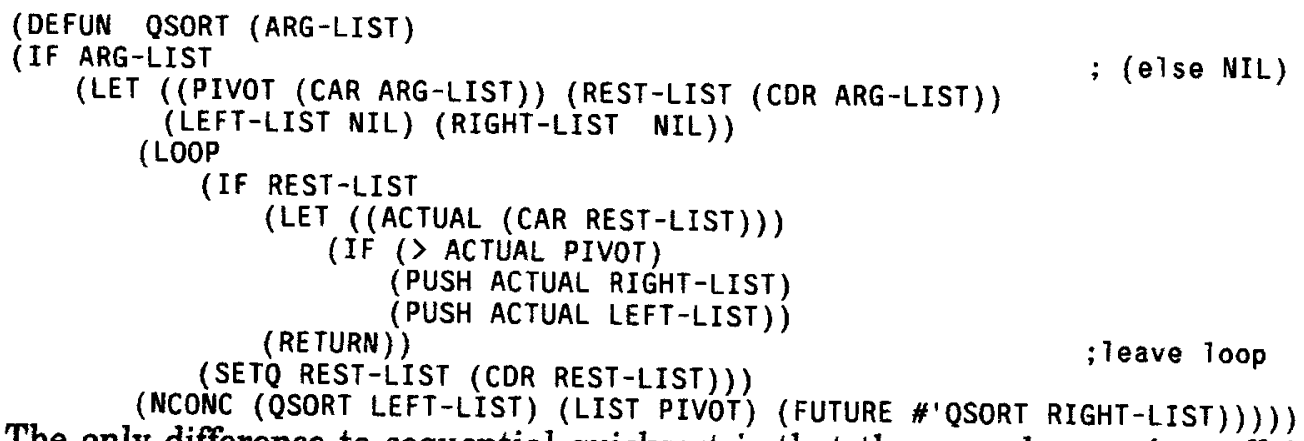

The only difference to sequential quicksort is that the second recursive call is done in parallel using the future construct; left and right lists are sorted in parallel. To understand the semantics of futures, consider the call (FUTURE function-name arguments). The future behaves almost like the Common Lisp construct funcall. The only difference is that it returns immediately after the evaluation of the arguments - with some place-holder value. This placeholder stands for a result which is not yet computed. Eventually, the function denoted by function-name is applied asynchronously to the evaluated arguments. 
When it returns the place-holder is overwritten with the real result. Placeholders are first class citizens : they may be copied and passed as argument to functions like all other values. Further, they may be used to build other values, e.g. as argument of the list-creating function CONS. Obviously, this black box treatment stops inside primitive constructs which "really need their arguments" like arithmetic operations. This is what we mean by touching. According to the semantics of future-based languages an implicit wait for the argument values has to be done in such circumstances: The evaluation is deferred until the argument value is accessible. In the above example the implicit wait is done for left lists but not for right lists. The reason for this is the fact that NCONC is not symmetric with respect to its arguments: the last one is handled in black box fashion.

There are two properties which are necessary for implicit waiting for the $i^{\prime}$ th argument of a construct call $(F \ldots X \ldots)$.

- the i'th argument position must be touching

- the construct $F$ must be primitive

The properties with respect to touching result directly from the (sequential) semantics of predefined LISP constructs. In contrast, it is up to the language designer to classify constructs as primitive or non-primitive (with implications on the obtainable amount of parallelism).

\section{Checks : The Price for Implicit Synchronization}

To understand the aim of touching analysis it is necessary to have a look on some implementation aspects. As in sequential LISP objects are represented by tagged cells. Future objects are represented by cells with a special tag value. Further, a future cell contains a pointer to a data structure representing the "content" of the future. Hence there are 3 different kinds of objects to be considered:

- undetermined future objects can not be accessed immediately. The evaluation has to be suspended until the objects become determined.

- The values of determined objects can be read and written without any synchronization but they may have to be accessed through a chain of pointer dereferencing actions.

- present objects are conventional LISP objects. Of course, every present object is determined.

Given these three kinds of objects, WAIT (explicit and implicit) can be defined:

WAIT $(X)=$

if is-present $(X)$ then $X$

else

if future-is-not-computed $(X)$ then dowait $(X)$ endif

WAIT (deref $(X))$

:this check occurs very often

; and fails rarely

; the evaluation is suspended

; follow dereferencing chain

endif

The check in the first line is the point of interest. The aim its elimination. Provided we know at compile time that $X$ is present the code simply becomes WAIT $(X)=X$. Less important, when we know that $X$ is determined there is no need for 
the check future-is-not-computed inside the recursion. In this case the check will fail for every recursion step and hence can be eliminated.

\section{Touching Analysis : An Example}

Consider the function MAP-DESTRU which performs a modification of its first argument (a list) by applying its second argument (a functional object) to each element of the list.

We first mark the touching positions with question marks.

(DEFUN MAP-DESTRU (SPINE ACTION)

(LET ( (ACTLIST SPINE) (LOOP

(ACTUAL NIL) (RESULT NIL))

(I

(? (NULL (? ACTLIST)))(RETURN))

(SETQ ACTUAL (CAR (? ACTLIST)))

(RPLACA (? ACTLIST)(FUNCALL (? ACTION) ACTUAL))

(SETQ ACTLIST (CDR (? ACTLIST)))) SPINE) )

Obviously $C A R, N U L L$ and $C D R$ touch their arguments. IF touches always its first argument, the touching of the second and third depending on the context (in this example, it does not touch). Note that SETQ (assignment) overwrites its first argument rather than touching it.

Applying the analysis we get some positions where we know that arguments are already determined (marked by "!") respectively present (marked by "\$").

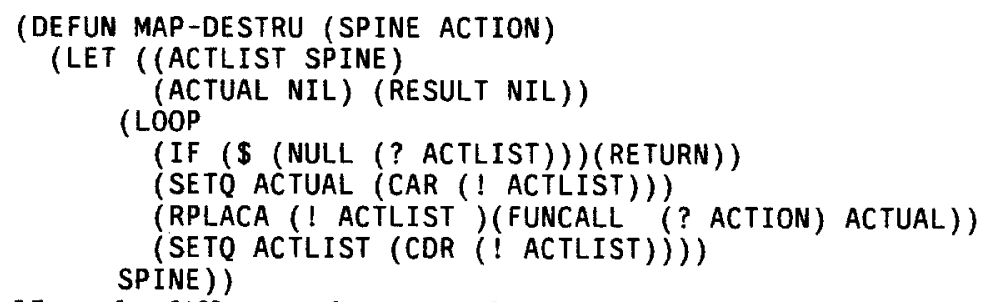

Note the difference between determined and present.: the function NULL yields a present value. In contrast, it touches its argument making it determined.

\section{Introducing Waits: The Assignment Paradigm}

We can go one step further to obtain more present objects: for every argument on a touching position a WAIT will be performed anyhow. Why not perform the WAIT explicitly and overwrite the argument (in case it is a variable) with the result value? (It is crucial to consider the exact definition of the semantics in order to allow such transformations. However, the rationale for this is beyond the scope of this paper.)

(DEFUN MAP-DESTRU (SPINE ACTION)

(LET ( (ACTLIST SPINE)

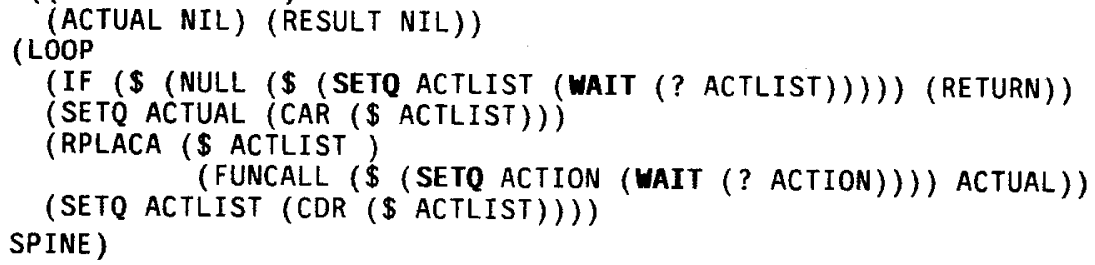

We gain present values rather than determined ones. Note that there are more 
present objects (and are indeed recognized by the analysis) than those marked but we do not bother about them because they do not occur on touching positions.

\section{The Semantics of Touching Analysis}

We now give somewhat simplified definitions for touching, strictness, implicit wait, present, determined and primitive in order to come to a more exact statement of the touching problem.

Every data object is uniquely determined by a reference.

Moreover, any object may have a contents. While the reference of an object exists during its lifetime the contents may be - at least initially - undefined. This is the typical situation when an object is created by a future function call.

\section{Definition}

An object is called determined iff it has a defined contents. A variable (or parameter) is determined iff its value is a determined object.

\section{Definition}

An object is called present iff it is determined during its entire lifetime. A variable is present iff its value is a present object. A variable or parameter is not present iff it is bound either to an object created by a future call or received its value from a variable or parameter which is not present either.

Obviously any present object variable or parameter is determined.

Note that both definitions ignore the structure of objects. Present or determined lists for instance may contain elements which have arbitrary properties.

\section{Definition}

A construct application $\left(F X_{1} \ldots X_{i-1} X i X_{i+1} \ldots X_{n}\right)$ is called i-touching iff for any $\mathrm{Y}$ (especially for any undetermined $\mathrm{Y}$ ), $\mathrm{Y}$ would be determined after the application $\left(F X_{1} \ldots X_{i-1} Y X_{i+1} \ldots X_{n}\right)$. In other words, the contents of the object resulting from the evaluation of the argument is needed during the function application. A construct $F$ is called i-touching iff every application of $F$ is $i$ touching.

\section{Observation}

In EDS LISP every $i$-touching construct is $i$-strict. The reverse is not true. In LISP all functions are strict (even functions like CONS !). Obviously, not all functions are touching (e.g. CONS).

\section{Definition}

A function application is called i-result-touching for some $i$ iff its $i$ th result is determined. A function is called i-result-touching iff all its applications are $i$ result-touching.

The semantics definition of EDS LISP contains a specification of all primitive functions with respect to the properties touching and result-touching.

\section{Examples}

- the functions $C A R, C D R$, etc. are touching and not result-touching.

- +,/,-, etc. are touching w.r.t. all arguments and result-touching.

- the special form SETQ (assignment) is neither touching nor result-touching.

- the function RPLACA is 1-touching and result-touching. 
- the special form IF is 1-touching and not result-touching.

- the functions CONS and LIST are not touchingbut result-touching.

- the function FUTURE is 1-touching and not result-touching.

- the function WAIT is touching and result-touching.

Note that these properties yield in any context. Special calls like (CAR (IF ...)) lead to touching of more arguments (of $I F$ ). But these are properties of the call context rather than properties of $I F$.

\section{The semantics of EDS LISP with respect to implicit synchronization}

- WAIT leads to synchronization iff its argument is not determined: The evaluation is suspended until its argument receives a value. This happens when the return value of a function spawned by a future becomes determined.

- The object returned by WAIT is present and has the same value as the argument of WAIT.

- For every touching position of a primitive construct an implicit WAIT occurs. No implicit WAIT occurs on other positions.

These assertions together with the touching property definitions of all primitive functions are part of the semantic definition for EDS LISP. Further, they are the basis of the touching analysis.

\section{The Touching Analysis Scenario}

Given a set of functions find those function applications $\left(\mathbf{F} \ldots \mathbf{X}_{\mathbf{i}} \ldots\right)$ which obey the following conditions:

a) $\mathrm{F}$ is $i$-touching and primitive.

b) $\mathrm{X}_{\mathrm{i}}$ is determined or present (in the context where $(F \ldots X i \ldots$ ) occurs).

The second condition holds in two typical situations:

1) $X_{i}$ is touched by some other touching function or

2) $X_{i}$ stems from a result-touching function application (including constants).

\section{Abstract Interpretation}

Abstract interpretation simulates the run time behaviour of programs in a simpler domain. For touching analysis this domain is three valued and totally ordered: ? $<!<\$$, in words: no-prop < determined < present. Properties of locals and parameters w.r.t. this domain are collected by the abstract interpretation algorithm. Worst case approximations are made for unknown data, conditionals and loops. It should be noted that there is no reason to restrict the analysis to purely functional programs. Loops and multiple assignments are handled in a straightforward manner. To obtain good results the analysis infers properties of user defined functions. This leads to intraprocedural optimization.

Recursion is handled in a way which is well known from strictness analysis: 
- We start with an optimistic (!) assumption, namely that all arguments of the recursive calls are touched and all results are determined resp. present.

- The propagation is done iteratively. Every iteration computes new properties and new function bodies using the propagation algorithm of the nonrecursive analysis for every step.

- The new property approximation is obtained by join between the old one and the properties obtained by the propagation.

- The iteration stops iff the function properties do not change any more.

Note that the start assumptions are optimistic rather than pessimistic. Although recursion and loops are treated very similar this is a fundamental difference.

\section{Implementation Results and Related Work}

The algorithm is implemented in Common LISP working by source to source transformation. It covers a functionality of over 100 LISP constructs including all aspects described in this document. Moreover special (dynamic) variables and closures are handled.

The identification of present with determined enforced by the assignment paradigm is optional. However, we feel that it is almost always useful.

Some benchmarks have been run before and after the analysis. We have counted the occurring touching positions and the present respective determined arguments on such positions. Both benchmark tables give ratios relative to the total

\begin{tabular}{|c|c|c|c|c|}
\hline Check elimination ratios w.r.t. touching positions & 1 & 2 & 3 & 4 \\
\hline without assignment paradigm : present's & 0.17 & 0.16 & 0.14 & 0.59 \\
without assignment paradigm : determined's & 0.59 & 0.67 & 0.57 & 0.15 \\
\hline with introduced assignments : present's & 0.67 & 0.83 & 0.71 & 0.84 \\
\hline
\end{tabular}

1: MAP-DESTRU, 2: as MAP-DESTRU but with the first loop traversal unrolled, 3: non-destructive MAP, 4: DESTRUCTIVE benchmark (Gabriel benchmark)

number of touching positions. The rows in the first table show the elimination ratios for the analysis with and without introduced assignments, respectively.

\begin{tabular}{|c|c|c|c|c|}
\hline Check elimination ratios w.r.t. touching positions & TAK *) & QSORT & FAC & FIB \\
\hline non-recursive (naive) analysis & 0.33 & 0.64 & 0.66 & 0.77 \\
\hline recursive analysis & 0.60 & 0.67 & 0.79 & 0.84 \\
\hline
\end{tabular}

Some well-known recursive functions $\left({ }^{*}\right)$ : a Gabriel benchmark)

The second benchmark table shows results of the naive, non-recursive analysis versus the recursive analysis (the version with introduced assignments only).

All ratios show a significant improvement of the run time behavior (of course depending on the cost of these checks on an individual machine). 
Touching analysis has been done in the Mul-T project in the context of a compiler for Scheme ([KrHaMo89]). The exact definition of the touching scenario, however, is lacking. Moreover, it seems that they analyze functional constructs, only. Although the touching analysis problem is different to the strictness analysis problem (see section 4), the method is very similar. With respect to the method we are very close to [CIPJo86]. However, there is no reason to restrict the method to functional programs.

Another related topic is type inference (see e.g. [PJo87], chapter 8 and 9).Touching analysis is a special case of type inference. Our algorithm is nicely extendible to type inference. The inference methods used in the context of functional languages do not fit for LISP. Note that LISP is neither functional nor type-safe. Hence properties are different on different program locations. This is in sharp contradiction to the concept of type in statically typed languages.

Methods used in some compiler optimizers are related to touching analysis, too. Lifetime analysis - albeit more simple - uses similar ideas (see e.g. [AhSeUl86]).

Due to page limitations, some of the aspects discussed in this paper are somewhat simplified. A more accurate framework with the formal justification w.r.t. recursive analysis for imperative languages is presented in a forthcoming paper. The extension to non-LISP future-based languages is subject of further work.

\section{Acknowledgments}

I wish to thank T. Henties, H. Ilmberger and M. Reich for fruitful discussions about strictness and touching and for proofreading earlier versions of this paper. This work was sponsored by the EC within project ESPRIT EP 2025.

\section{References}

[AbHa 87]

[AhSeU186]

[ClPJo86]

[HaHe 90]

[Hal85 ] R. Halstead: Multiplisp: A Language for Concurrent Symbolic
Computation, in ACM Transactions on Programming Languages
and Systems, October 1985.

[KrHaM089] D. Kranz, R. Halstead, Jr., E. Mohr: Mul-T: A High-Performance

S. Abramsky, C. Hankin, editors: Abstract Interpretation of Declarative Languages, Ellis Horwood Series in Computers and Their Applications, 1987.

A. Aho, R. Sethi, J. Ullmann, Compilers: Principles, Techniques, and Tools, Addison-Wesley, 1986.

C.Clack, S. Peyton-Jones: Strictness Analysis: a Practical Approach, in Proceedings of the ACM Conference on Functional Languages and Computer Architecture 1985, LNCS 201.

C. Hammer, T. Henties: Parallel Lisp on a Distributed Machine, in EUROPAL Workshop on High Performance and Parallel Computing in Lisp, London, 1990. and Systems, October 1985. Parallel Lisp, in ACM Programming Language Design and Implementation, Portland, Oregon, June 1989.

[PJoC187] S.Peyton-Jones, C.Clack: Finding Fixpoints in Abstract Interpretation, in [AbHa87].

[PJo87] S.Peyton-Jones, editor: The Implementation of Functional Programming Languages, Prentice Hall, 1987. 